\title{
Ongoing Improvement in Acute Ischemic Stroke Therapy Per Concurrent Guidelines and Easily Implementable Quality Improvement Protocol
}

Shimeng Liu ${ }^{1,3}$, Zhu Zhu¹, Mohammad Shafie ${ }^{1}$, Hermelinda Abcede ${ }^{1}$, Jay Shah ${ }^{1}$, Shuichi Suzuki², Li-Mei Lin², Kiarash Golshani², Dana Stradling ${ }^{1}$, Wengui Yu ${ }^{1^{*}}$

${ }^{1}$ Department of Neurology, University of California, Irvine, CA, USA

${ }^{2}$ Department of Neurosurgery, University of California, Irvine, CA, USA

${ }^{3}$ Tiantan Hospital, Beijing, China

*Corresponding Author: Wengui Yu, Department of Neurology, University of California, Irvine, CA, USA

Received date: May 03, 2021; Accepted date: May 20, 2021; Published date: May 27, 2021

Citation: Shimeng Liu, Zhu Zhu, Mohammad Shafie, Hermelinda Abcede, Jay Shah, Shuichi Suzuki, Li-Mei Lin, Kiarash Golshani, Dana Stradling, Wengui Yu., (2021) Ongoing Improvement in Acute Ischemic Stroke Therapy Per Concurrent Guidelines and Easily Implementable Quality Improvement Protocol. J. Neuroscience and Neurological Surgery. 9(1); DOI:10.31579/2578-8868/184

Copyright: () 2021 Wengui $Y u$, This is an open-access article distributed under the terms of The Creative Commons Attribution License, which permits unrestricted use, distribution, and reproduction in any medium, provided the original author and source are credited

\section{Abstract:}

Background: Despite proven efficacy of intravenous tissue plasminogen activator (tPA) and endovascular thrombectomy (EVT) in acute ischemic stroke, there has been slow administration of these therapies in the real world practice. We examined the ongoing quality improvement in acute stroke care at our comprehensive stroke center.

Methods: Consecutive patients with acute ischemic stroke from 2013 to 2018 were studied. Patients were managed using Code Stroke algorithm per concurrent AHA guidelines and a simple quality improvement protocol implemented in 2015. Demographics and clinical data were collected from Get-With-TheGuideline-Stroke registry and electronic medical records. Patients were divided into 3 groups per admission and implementation date of quality improvement initiatives. Quality measures, including rates of intravenous tPA and EVT, door-to-needle (DTN) time, and door-to-puncture (DTP) time, were analyzed with general mean linear regression models and Jonckheere-Terpstra test.

Results: Of the 1,369 eligible patients presenting within 24 hours of symptom onset or wakeup stroke, the rate of intravenous tPA was 20\%, 30\% and 22\%, respectively, in 2013-2014, 2015-2016, and 2017-2018. In contrast, EVT rate was $9 \%, 14 \%$ and $15 \%$, respectively. Based on Jonckheere-Terpstra test, there was significant ongoing improvement in the median DTN time $(57,45,39$ minutes; $p<0.001)$ and DTP time $(172,130,114$ minutes; $p=0.009)$ during the 3 time periods, with DTN time $\leq 60$ and $\leq 45$ minutes in $80 \%$ and $63 \%$ patients, respectively, in 2017-2018.

Conclusions: Getting with the guidelines and simple quality improvement initiatives are associated with satisfactory rates of acute stroke therapy and ongoing improvement in door to treatment times.

Keywords: acute ischemic stroke; benchmarks; endovascular thrombectomy; intravenous thrombolysis; outcomes; quality improvement

Running Title : Recurrent Hemorrhagic Transformations

\section{Introduction}

Stroke is the $5^{\text {th }}$ cause of death and a leading cause of long-term disability in the United States [1,2]. Intravenous thrombolysis with tissue plasminogen activator (tPA) is the only proven medical therapy for acute ischemic stroke (AIS) within 4.5 hours of symptoms onset [3, 4]. The benefit of tPA is time-dependent, with better outcome from earlier treatment $[5,6]$. In patients with AIS from large vessel occlusion (LVO), endovascular thrombectomy (EVT) within 6 to 24 hours of last-knownwell (LKW) has become the standard of care since 2015 [2,7-13]. EVT is also time sensitive. Every 1-hour delay from LKW to arterial puncture was associated with more severe disability [14].
The American Heart Association (AHA)'s Get with the Guidelines (GWTG)-Stroke programme was established in 2003 to collect data on patient characteristics, hospital adherence to guidelines and inpatient outcomes [15]. For time-sensitive quality measures in stroke care, American Heart Association /American Stroke Association (AHA/ASA) launched the Target: Stroke initiative in January 2010 [16]. Two years later, the Joint Commission started comprehensive stroke center (CSC) certification [17]. Despite these initiatives, only $50 \%$ of AIS patients registered in GWTG-Stroke from October 2012 to April 2015 received intravenous tPA within 60 minutes of emergency room arrival [18]. 
In a study of patients treated at 134 CSCs and 1047 primary stroke centers (PSCs) in the United States from 2013 to 2015, the median door-to-needle (DNT) times were 52 and 61 minutes, respectively [19]. The rate of intravenous tPA was $14.3 \%$ at CSCs and $10.3 \%$ at PSCs, while the EVT rate was only $4.1 \%$ at CSCs and $1.0 \%$ at PSCs, respectively [19]. In a most recent study, Menon et al. analyzed data from 195 CSCs and identified 2929 patients treated with EVT from October 2014 to September 2016. The median annual EVT volume per center was 16 and the median door to first pass time was 130 minutes [20].

Benchmarking is critical for quality improvement in stroke care. Our stroke center received CSC certification in 2013 and implemented a simple quality improvement protocol in January 2015 [21]. The aim of this study was to investigate the evolution of stroke care and ongoing improvement in quality indicators since CSC certification.

\section{Materials and Methods}

Consecutive patients with AIS admitted at a 417-bed comprehensive stroke center in California, USA, from January 1, 2013 to December 31, 2018 were included in the study. The patient list was generated from the prospectively maintained AHA/ASA GWTG-Stroke Registry at our hospital. The registry uses a web-based patient management tool to collect clinical data on consecutively admitted patients, to provide decision support, and to enable real-time online reporting [22]. Patients with TIA, stroke mimics, subacute stroke, inpatient stroke and brain hemorrhage were excluded. Patients who were transferred from outside facilities were also excluded. The screening flow chart for eligible patients is shown in Figure 1.

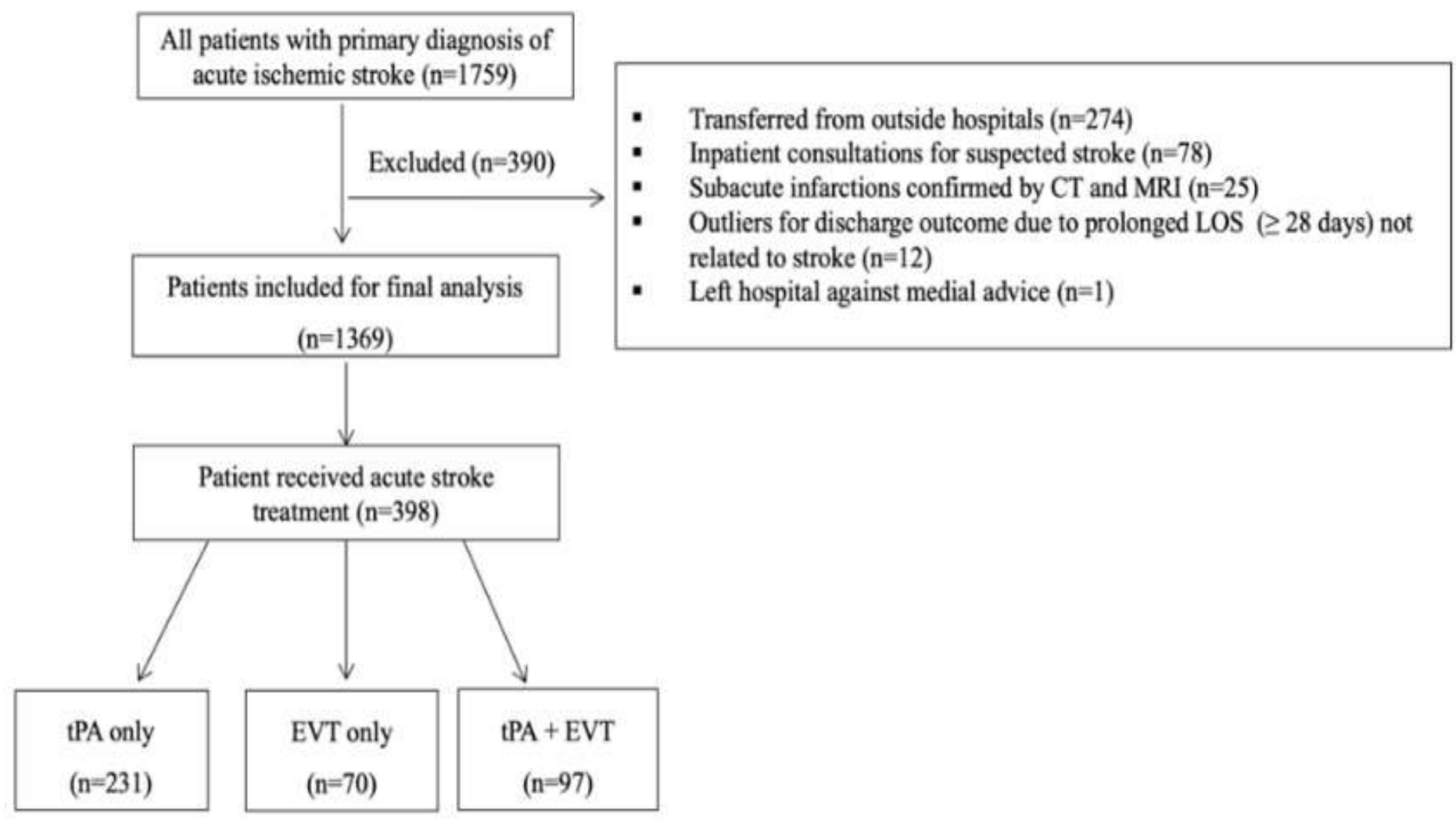

From January 1, 2013 to December 31, 2018, 1759 patients were admitted to the medical center for acute ischemic stroke AIS. After excluding ineligible patients ( $n=390), 1369$ patients were included in final analysis.

Figure 1: Screening of eligible patients for the study

All patients were managed by the emergency department (ED) and stroke team using standard ED code stroke algorithm per concurrent AHA guidelines (Figure 2). 


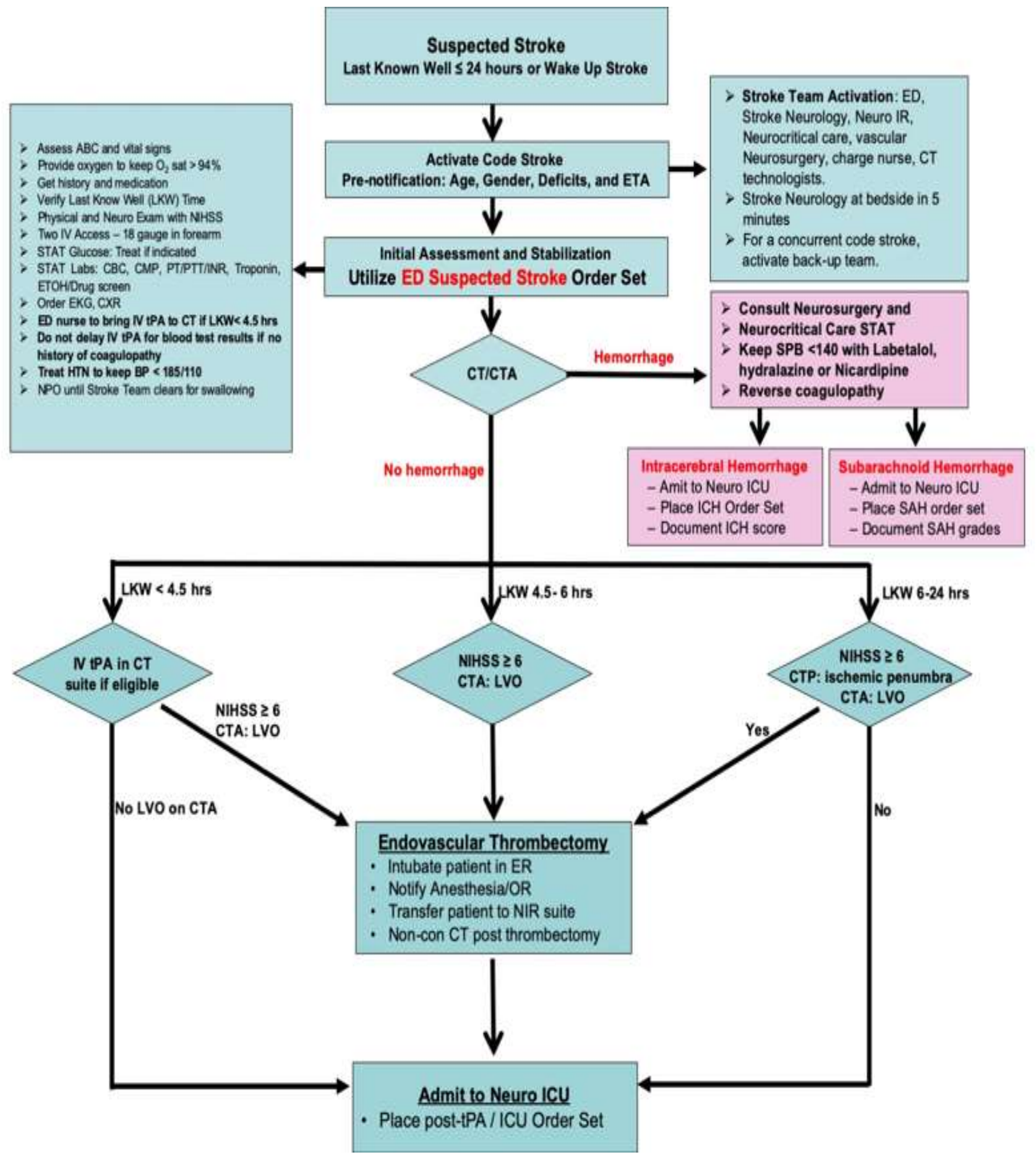

Abbreviations: ETA: estimated time of arrival; ED: emergency department; CT: Computerized Tomography; CTA: Computerized Tomography Angiography; IR: interventional radiology; NIR: neuro interventional radiology; ABC: airway, breath and circulation; NIHSS: National Institutes of Health Stroke Scale; IV: intravenous; CBC: complete blood count; CMP: comprehensive metabolic panel; PT: prothrombin time; PTT: partial thromboplastin time; INR: international normalized ratio; ETOH: alcohol; EKG: electrocardiogram; CXR: chest X-ray; tPA: tissue plasminogen activator; LKW: last-known well; HTN: hypertension; NPO: nothing by mouth; LVO: large vessel occlusion; OR: operation room; Non-con CT: noncontrast CT; ICU: intensive care unit.

Figure 2: Emergency Department Code Stroke Algorithm 
A simple quality improvement protocol was implemented in January 2015 to minimize delays in DTN time for tPA as previously described [21]. It allowed stroke team to treat hypertension in the emergency room to keep blood pressure (BP) $<185 / 110 \mathrm{mmHg}$, to give tPA before getting blood test results unless patients were taking anticoagulants, and to give tPA in the CT scan suite. To continuously improve stroke care, we update our ED Code Stroke Algorithm and order sets annually per guidelines and advances in the field. In addition, we have a weekly stroke quality improvement committee meeting and case conferences to review every stroke admission for ongoing quality improvement.

The following data, including patient demographics, co-morbidities, National Institutes of Health Stroke Scale (NIHSS), BP, LKW-to-door time, door-to-imaging time, DTN time for tPA, door-to-puncture (DTP) time for EVT, symptomatic intracranial hemorrhage (sICH), length-ofstay (LOS) in the Intensive Care Unit (ICU) or hospital, and modified Rankin Scale (mRS) at hospital discharge, were collected from the registry and electronic medical record by experienced neurologists.[21] Laboratory results, including comprehensive metabolic panel, fasting lipid profile, and glycol-hemoglobin A1c (HbA1c), were also collected. sICH was defined as intraparenchymal hematoma, subarachnoid hemorrhage, or intraventricular hemorrhage associated with a worsening of the NIHSS score by $\geq 4$ points within $24 \mathrm{~h}$ of tPA and/or EVT [5]. A mRS score of 0-3 at discharge was defined as favorable outcome. NIHSS, sICH and mRS were estimated by the stroke team. Uncertain cases or disagreement were adjudicated by experienced neurologists.

To investigate the ongoing quality improvement in acute stroke therapy, patients were categorized into three groups according to admission and implementation date of quality improvement initiatives [16-21]: January 1, 2013 to December 31, 2014; January 1, 2015 to December 31, 2016; and January 1, 2017 to December 31, 2018. Time sensitive-quality indicators, including LKW-to door time, DTI time, DTN time, DTP time, rates of tPA and EVT, sICH rate, LOS in the ICU and hospital, and functional outcome at hospital discharge, were analyzed and compared among the three time periods.

Statistical analysis
Categorical variables were expressed as frequencies and percentages (\%), and continuous variables as median and interquartile range (IQR). General linear means regression models and Jonckheere-Terpstra test were used to assess the estimated difference of continuous variables. Univariate logistic regression analysis was performed to test the crude ORs $(95 \% \mathrm{CI})$ of quality benchmarks between groups. Multivariate logistic regression models were performed to determine the adjusted ORs (95\% CI) of favorable outcomes (mRS score 0-3) at hospital discharge, in association with the admission time. In the multivariate models, we adjusted age, initial NIHSS scores, and DTN time to exclude the potential confounding factors. The SAS statistical software (version 9.4; SAS Institute, Cary, NC, USA) was used to perform the data analysis.

\section{Results}

From January 1, 2013 to December 31, 2018, 1,759 patients were admitted to the medical center for AIS within 24 hours of symptom onset or wakeup stroke. During the initial screening, 390 patients were excluded from the study for the following reasons (Figure 1): 1). 274 patients were transferred from outside hospitals for higher level of care, including 58 treated with intravenous IPA at outside facilities; 2). 78 patients were inpatient consultations for suspected ischemic stroke; 3). 25 patients presented with subacute infarction that was confirmed by brain imaging, 4). 12 patients were outliners for LOS ( $\geq 28$ days) and discharge outcome due to insurance issue; and 5). 1 patient left hospital against medical advice.

A total of 1,369 patients were included in the final analysis. Of note, 53 patients had 2 admissions, 3 patients had 3 admissions, and 1 patient had 4 admissions during the study period. The median age and interquartile range (IQR) of the patients was 71 (24). There were 755 men and 614 women. There were 52\% White, 23\% Asian, 18\% Hispanic, $2 \%$ American African, and 5\% other ethnic patient population. Of the entire cohort, 71\% listed English as primary language, 16\% spoke Spanish, and 12\% spoke other languages. The most common past medical histories were hypertension (71\%), diabetes mellitus (36\%), and hyperlipidemia (39\%). The baseline demographics and clinical data of the 3 groups were shown in Table 1. 


\begin{tabular}{|l|c|c|c|}
\hline \multicolumn{1}{|c|}{ Variables } & $\mathbf{2 0 1 3 - 2 0 1 4}$ & $\mathbf{2 0 1 5 - 2 0 1 6}$ & $\mathbf{2 0 1 7 - 2 0 1 8}$ \\
\hline Numbers of patients & 454 & 451 & 464 \\
\hline Age, median (IQR) & $69(23)$ & $73(23)$ & $71(23)$ \\
\hline Female (\%) & $220(48.5)$ & $196(43.5)$ & $198(42.7)$ \\
\hline Race & & & \\
\hline White (\%) & $240(52.9)$ & $263(56.1)$ & $216(46.6)$ \\
\hline Asian (\%) & $96(21.1)$ & $95(21.1)$ & $123(26.5)$ \\
\hline Hispanic (\%) & $82(18.1)$ & $72(16.0)$ & $86(18.5)$ \\
\hline African American (\%) & $13(2.9)$ & $7(1.6)$ & $13(2.8)$ \\
\hline Others (\%) & $23(5.1)$ & $24(5.3)$ & $26(5.6)$ \\
\hline Preferred language & & & \\
\hline English (\%) & $307(67.6)$ & $325(72.1)$ & $345(74.4)$ \\
\hline Spanish (\%) & $96(21.1)$ & $67(14.9)$ & $62(13.4)$ \\
\hline Other language (\%) & $51(11.2)$ & $59(13.1)$ & $55(11.9)$ \\
\hline Hypertension (\%) & $344(75.8)$ & $323(71.6)$ & $308(66.4)$ \\
\hline Diabetes (\%) & $189(41.6)$ & $156(34.6)$ & $152(32.8)$ \\
\hline Hyperlipidemia (\%) & $175(38.5)$ & $178(39.5)$ & $187(40.3)$ \\
\hline Previous antithrombotic use (\%) & $237(52.2)$ & $193(42.8)$ & $199(42.9)$ \\
\hline Obesity (\%) & $109(24)$ & $9(2.0)$ & $71(15.3)$ \\
\hline SBP, mmHg, median (IQR) & $159(43)$ & $163(44)$ & $162(47)$ \\
\hline DBP, mmHg, median (IQR) & $86(24)$ & $88(23)$ & $89(23)$ \\
\hline NIHSS, median (IQR) & $5(12)$ & $6(13)$ & $5(10)$ \\
\hline LDL-c, mg/dl, median (IQR) & $2.53(1.40)$ & $2.45(1.40)$ & $2.33(1.16)$ \\
\hline Hb A1c, \%, median (IQR) & $6.0(2.2)$ & $6.0(1.3)$ & $5.9(1.4)$ \\
\hline LKW-to door time, median (IQR) & $420(1745)$ & $307(1063)$ & $288(824)$ \\
\hline
\end{tabular}

Abbreviations: $D B P$, diastolic blood pressure; Hb Alc, hemoglobin A1c; IQR, interquartile range; LDL-c, low-density lipoprotein cholesterol; LKW, last known well; NIHSS, National Institutes of Health Stroke Scale; SBP, systolic blood pressure.

Table 1: Demographics and clinic data of patients from the 3 time period

There were no significant differences among the 3 groups in age, gender, race, preferred language, major co-morbidities, initial BP, NIHSS scores, LDL and $\mathrm{Hb}$ A1c levels.
Ongoing improvement in treatment rate and quality benchmarks A total of 328 patients received intravenous tPA in the cohort (Table 2). 


\begin{tabular}{|l|c|c|c|c|}
\hline \multicolumn{1}{|c|}{ Variable } & $\mathbf{2 0 1 3 - 2 0 1 4}$ & $\mathbf{2 0 1 5 - 2 0 1 6}$ & $\mathbf{2 0 1 7 - 2 0 1 8}$ & $p^{\dagger}$ \\
\hline $\mathrm{n}$ & 454 & 451 & 464 & - \\
\hline tPA, n (\%) & $89(20 \%)$ & $136(30 \%)^{*}$ & $103(22 \%)$ & - \\
\hline DTI time, min, median (IQR) & $14(12)$ & $16(10)$ & $19(19)^{*}$ & 0.283 \\
\hline DTN time, min, median (IQR) & $57(31)$ & $45(29)^{*}$ & $39(30)^{*}$ & $<0.001$ \\
\hline DTN time $\leq 60$ min, $\mathrm{n}(\%)$ & $52(58 \%)$ & $100(74 \%)^{*}$ & $82(80 \%)^{*}$ & - \\
\hline DTN time $\leq 45$ min, $\mathrm{n}(\%)$ & $19(21 \%)$ & $70(51 \%)^{*}$ & $65(63 \%)^{*}$ & - \\
\hline DTN time $\leq 30$ min, $\mathrm{n}(\%)$ & $4(5 \%)$ & $30(22 \%)^{*}$ & $31(30 \%)^{*}$ & - \\
\hline sICH (\%) & $1(1.1)$ & $2(1.5)$ & $2(1.9)$ & - \\
\hline ICU LOS, median (IQR) & $3(3)$ & $2(2)$ & $2(2)$ & 0.751 \\
\hline Hospital LOS, median (IQR) & $6(7)$ & $3(4)$ & $3(3)$ & 0.120 \\
\hline Favorable outcome (\%) & $35(39.3)$ & $76(55.9)^{*}$ & $44(42.7)$ & - \\
\hline In-hospital mortality (\%) & $6(6.7)$ & $10(7.4)$ & $4(3.9)$ & - \\
\hline EVT, n(\%) & $39(9 \%)$ & $62(14 \%)^{*}$ & $66(15 \%)^{*}$ & - \\
\hline DTP time, min, median (IQR) & $172(71)$ & $130(67)^{*}$ & $114(65)$ & 0.009 \\
\hline sICH (\%) & $0(0 \%)$ & $1(1.6 \%)$ & $3(4.1 \%)$ & - \\
\hline ICU LOS, median (IQR) & $4(6)$ & $3(3)$ & $3(3)$ & 0.175 \\
\hline Hospital LOS, median (IQR) & $6(8)$ & $5(5)$ & $4(7)$ & 0.276 \\
\hline Favorable outcome (\%) & $10(26 \%)$ & $19(31 \%)$ & $23(35 \%)$ & - \\
\hline In-hospital mortality (\%) & $8(21 \%)$ & $11(18 \%)$ & $8(12 \%)$ & - \\
\hline
\end{tabular}

Abbreviations: DTI, door-to-image; DTN, door-to-needle; DTP, door-to-puncture; EVT, endovascular thrombectomy; ICU, intensive care unit; IQR, interquartile range; LOS, length of stay; NIHSS, National Institutes of Health Stroke Scale; sICH, symptomatic intracranial hemorrhage.

* Difference was significant when compared with 2013-2014: univariate logistic regression models were used to compare categorical variables; general linear means regression models were used to compare continuous variables.

$\dagger$ Trend analysis with Jonckheere-Terpstra test.

Table 2: Quality indicators and outcomes of the patients during the 3 time periods

The tPA rate was $20 \%, 30 \%$ and $22 \%$ in $2013-2014,2015-2016$, and 2017-2018, respectively (Table 2). In the crude logistic regression model, tPA rate was significantly higher in 2015-2016 than that in 2013-2014 (OR, 1.77; 95\% CI, 1.30-2.40; $p=0.0003$ ). There was no significant difference in tPA rate between 2017-2018 and 2013-2014 (OR: 1.17; 95\% CI: $0.85-1.61 ; p=0.33)$. There was no significant difference in DTI time among the 3 time periods. However, there was significant ongoing improvement in median DTN time (57, 45, and 39 minutes, respectively) during the 3 groups per trend analysis, with DTN time $\leq 60,45$ and 30 minutes in $80 \%, 63 \%$ and $30 \%$ of patients, respectively, during 2017 2018. There was no significant difference in sICH rate, LOS in the ICU or hospital, and mortality at hospital discharge among the 3 groups. Of note, significantly more patients had favorable outcomes (mRS score 03 ) at hospital discharge in 2015-2016 than in 2013-2014. There was no difference between 2013-2014 and 2017-2018. (Table 2)

A total of 167 patients received EVT with or without intravenous tPA. The rate of EVT was $9 \%, 14 \%$ and $15 \%$, respectively, during the 3 time periods. Significantly more patients were treated with EVT in 2015-2016 
(OR, 1.70; 95\% CI, $1.11-2.59 ; p=0.01)$ and 2017-2018 (OR, 1.77; 95\% CI, $1.16-2.68 ; p=0.008$ ) than in 2013-2014, due to acceptance of EVT as standard of care for patients with AIS from LVO in 2015.

There was also continual improvement in median DTP time (172, 130, 114 minutes) during the 3 time periods per trend analysis with Jonckheere-Terpstra test. There was no significant difference in $\mathrm{sICH}$ rate, LOS in the ICU and Hospital, or favorable outcome among the 3 groups. There appeared to be a trend of decreased mortality rate at hospital discharge during the 3 time periods. The overall rates of sICH and mortality after EVT were $2.4 \%$ and $16 \%$, respectively.

\section{Multivariate logistic regression analysis of favorable outcomes with Wald Model}

To examine the predictor of favorable outcomes after intravenous tPA, we performed a multivariate logistic regression model with Wald ChiSquare test (Table 3). After adjusting for age, NIHSS scores and DTN time, age and initial NIHSS were independently associated with favorable outcomes at hospital discharge.

\begin{tabular}{|l|c|c|c|c|c|}
\hline & OR & 95\% CI & DF & Wald Chi-Square & $\boldsymbol{p}$ \\
\hline Year of admission & 1.32 & $0.91-1.92$ & 1 & 1.76 & 0.146 \\
\hline Age & 0.97 & $0.96-0.99$ & $\mathbf{1}$ & $\mathbf{9 . 7 1}$ & $\mathbf{0 . 0 3 5}$ \\
\hline Initial NIHSS scores & 0.87 & $0.83-0.91$ & $\mathbf{1}$ & $\mathbf{5 0 . 3 0}$ & $<\mathbf{0 . 0 0 1}$ \\
\hline DTN time & 0.99 & $0.98-1.00$ & 1 & 2.55 & 0.330 \\
\hline
\end{tabular}

Abbreviations: OR, odds ratio; CI, Confidence Interval; NIHSS, National Institutes of Health Stroke Scale.

Table 3: Multivariate regression model assessing the predictor of favorable outcomes after intravenous tPA therapy

\section{Discussion}

We have reduced the median DTP times from 172 minutes in 2013-2014 to 114 minutes in 2017-2018. The results were similar to the reported 130145 minutes at the CSCs registered in GWTG-Stroke program [19, 20], but very suboptimal compared to the median 47 minutes reported at highvolume center with standardized protocol and conscious sedation for EVT [23]. Of note, DTP times were reduced to 17 - 20.5 minutes when the patients were directly transferred from ED to angiography suite [24, 25]. The median annual EVT volume per CSC in the United States was 16 (IQR, 10-27) [20]. That was only slightly more than one EVT a month. Each 5-case increase in EVT volume per year was found to be associated with a $3 \%$ shorter door to first pass time, up to a case volume of 40 per year $(P<0.001)$ [20]. Currently, only a minority of CSCs in the United States are providing EVT to $>40$ patients per year partly due to very lenient criteria for CSC certification [17]. EVT is a labor-intensive procedure that requires a coordinated effort among numerous healthcare providers and timely access to angiography facilities. In addition to the Code Stroke team 24/7 in house, patients may also need intubation for the procedure, timely transport to the angiography suite, anesthesiologist, angiography suite nurses and technicians [24, 26]. Overnight and weekends pose additional challenges. Due to low EVT volume, it is financially impossible for individual CSC to implement rapid EVT protocol that requires the entire stroke team to be brought in for each possible code stroke [23].

Currently, three strategies, including transporting patients directly from emergency medical services to the CT scanner, conscious sedation, and transferring patients with suspected LVO from ER to angiography suite directly, were reported to have large independent effect on reducing door to treatment times [23-26].

Of note, significantly more patients were treated with iv tPA at our center in 2015-2016 due to the implementation of a simple quality improvement initiative in January of 2015 [20]. However, the release of the PRISMS Trial results at the International Stroke Conference in January of 2018 led to decreased treatment of minor non-disabling stroke with tPA at our center in 2017-2018. PRISMS is a randomized trial of tPA versus aspirin for patients with minor nondisabling stroke (NIHSS 1-5). The primary outcome was minimal or no neurologic deficit (mRS 0-1) at 90 days. The study was stopped early, after enrollment of 313 patients with median NIHSS 2 and median DTN time 2.7 hours. There was no significant difference in functional outcome at 90 days $(81.5 \%$ in the aspirin group and $78.2 \%$ of patients in the tPA group). Thrombolysis showed no benefit even after exclusion of stroke mimics. sICH rate was $3.2 \%$ with $\mathrm{PPA}$ and $0 \%$ with aspirin [28].

Our study has many limitations. First, this is a single center retrospective study. The data were collected from GWTG-Stroke Registry and chart review. There could be data collection bias. Second, there was no longterm follow up information. However, benchmarking is essential for continuous quality improvement in stroke care. To further improve the door to treatment times, we plan to implement new initiatives on transporting patients directly from emergency medical services to the CT scanner, conscious sedation, and transferring patients with suspected LVO from ER to angiography suite. We will also start long-term outpatient follow-up on all patients after hospital discharge.

\section{Conclusions}

We demonstrated satisfactory rates of acute stroke treatment and ongoing improvement in door to treatment times at our CSC. Our findings suggest that implementation of concurrent AHA guidelines and new quality improvement initiatives are essential for continuous quality improvement in acute stroke care. 


\section{Declarations}

Ethics approval and consent to participate: The study was approved by the Institutional Review Board (IRB) at University of California Irvine Comprehensive Stroke Center. No identifiable information was indicated in the retrospective database-based study, so informed consent was not applicable.

\section{Consent for publication: Not applicable.}

Competing interests: Dr. Yu has received compensation for activities with Stryker and Amgen as a scientific consultant. However, the activities are not related to this research project. Other authors have nothing to disclose.

\section{Funding: None.}

Authors' contributions: Concept and design: Liu, Yu. Acquisition, analysis, and interpretation of the data, Liu, Zhu, Stradling, Yu. Drafting of the manuscript: Liu. Critical revision of the manuscript for important intellectual content: Zhu, Shafie, Abcede, Shah, Suzuki, Li, Golsahni, Yu. Statistical analysis: Liu, Zhu, and Yu

Acknowledgements: We appreciate the support from the University of California Irvine Xiaoqi Cheng \& Dongmei Liao International Stroke Research Scholarship.

Availability of data and materials: Dr. Yu has full access to all of the data in the study and takes responsibility for the integrity of the data and the accuracy of the data analysis.

\section{References}

1. Benjamin EJ, Muntner P, Alonso A, Bittencourt MS, Callaway CW, Carson AP, et al. Heart Disease and Stroke Statistics-2019 Update: A Report From the American Heart Association. Circulation. 2019;139 10:e56-e528; doi: 10.1161/cir.0000000000000659.

2. Powers WJ, Rabinstein AA, Ackerson T, Adeoye OM, Bambakidis NC, Becker K, Biller J, Brown M, Demaerschalk BM, Hoh B, Jauch EC, Kidwell CS, Leslie-Mazwi TM, Ovbiagele B, Scott PA, Sheth KN, Southerland AM, Summers DV, Tirschwell DL. Guidelines for the Early Management of Patients With Acute Ischemic Stroke: 2019 Update to the 2018 Guidelines for the Early Management of Acute Ischemic Stroke: A Guideline for Healthcare Professionals From the American Heart Association/American Stroke Association. Stroke. 2019 Dec;50(12):e344-e418.

3. The National Institute of Neurological Disorders and Stroke rt-PA Stroke Study Group. Tissue Plasminogen Activator for Acute Ischemic Stroke. New England Journal of Medicine. 1995;333 24:1581-1588; doi: 10.1056/nejm199512143332401.

4. Hacke W, Kaste M, Bluhmki E, Brozman M, Davalos A, Guidetti $\mathrm{D}$, et al. Thrombolysis with alteplase 3 to 4.5 hours after acute ischemic stroke. The New England journal of medicine. 2008;359 13:1317-1329; doi: 10.1056/NEJMoa0804656.

5. Lees KR, Bluhmki E, von Kummer R, Brott TG, Toni D, Grotta $\mathrm{JC}$, et al. Time to treatment with intravenous alteplase and outcome in stroke: an updated pooled analysis of ECASS, ATLANTIS, NINDS, and EPITHET trials. The Lancet. 2010;375 9727:1695-1703; doi: 10.1016/S0140-6736(10)60491-6.
6. Emberson J, Lees KR, Lyden P, Blackwell L, Albers G, Bluhmki E, et al. Effect of treatment delay, age, and stroke severity on the effects of intravenous thrombolysis with alteplase for acute ischaemic stroke: a meta-analysis of individual patient data from randomised trials. Lancet (London, England). 2014;29 384:19291935; doi: 10.1016/S0140-6736(14)60584-5.

7. Berkhemer OA, Fransen PSS, Beumer D, van den Berg LA, Lingsma HF, Yoo AJ, et al. A Randomized Trial of Intraarterial Treatment for Acute Ischemic Stroke. New England Journal of Medicine. 2014;372 1:11-20; doi: 10.1056/NEJMoa1411587.

8. Campbell BCV, Mitchell PJ, Kleinig TJ, Dewey HM, Churilov L, Yassi N, et al. Endovascular Therapy for Ischemic Stroke with Perfusion-Imaging Selection. New England Journal of Medicine. 2015;372 11:1009-1018; doi: 10.1056/NEJMoa1414792.

9. Goyal M, Demchuk AM, Menon BK, Eesa M, Rempel JL, Thornton J, et al. Randomized Assessment of Rapid Endovascular Treatment of Ischemic Stroke. New England Journal of Medicine. 2015;372 11:1019-1030; doi: 10.1056/NEJMoa1414905.

10. Saver JL, Goyal M, Bonafe A, Diener H-C, Levy EI, Pereira VM, et al. Stent-Retriever Thrombectomy after Intravenous t-PA vs. tPA Alone in Stroke. New England Journal of Medicine. 2015;372 24:2285-2295; doi: 10.1056/NEJMoa1415061.

11. Jovin TG, Chamorro A, Cobo E, de Miquel MA, Molina CA, Rovira A, et al. Thrombectomy within 8 Hours after Symptom Onset in Ischemic Stroke. New England Journal of Medicine. 2015;372 24:2296-2306; doi: 10.1056/NEJMoa1503780.

12. Nogueira RG, Jadhav AP, Haussen DC, Bonafe A, Budzik RF, Bhuva P, et al. Thrombectomy 6 to 24 Hours after Stroke with a Mismatch between Deficit and Infarct. New England Journal of Medicine. 2017;378 1:11-21; doi: 10.1056/NEJMoa1706442.

13. Albers GW, Marks MP, Kemp S, Christensen S, Tsai JP, OrtegaGutierrez S, et al. Thrombectomy for Stroke at 6 to 16 Hours with Selection by Perfusion Imaging. The New England journal of medicine. 2018;378 8:708-718; doi: 10.1056/NEJMoa1713973.

14. Saver JL, Goyal M, van der Lugt A, Menon BK, Majoie CB, Dippel DW, et al. Time to Treatment With Endovascular Thrombectomy and Outcomes From Ischemic Stroke: A Metaanalysis. Jama. 2016;316 12:1279-1288; doi: 10.1001/jama.2016.13647.

15. Ormseth CH, Sheth KN, Saver JL, Fonarow GC, Schwamm LH. The American Heart Association's Get With the Guidelines (GWTG)-Stroke development and impact on stroke care. Stroke Vasc Neurol. 2017 May 29;2(2):94-105. doi: 10.1136/svn-2017000092 .

16. Fonarow GC, Smith EE, Saver JL, Reeves MJ, Hernandez AF, Peterson ED, et al. Improving door-to-needle times in acute ischemic stroke: the design and rationale for the American Heart Association/American Stroke Association's Target: Stroke initiative. Stroke. 2011;42 10:2983-2989; doi: 10.1161/strokeaha.111.621342.

17. Advanced certification comprehensive stroke centers. The Joint Commission.

https://www.jointcommission.org/certification/advanced certifica tion comprehensive stroke centers.aspx. Accessed 23 April 2019.

18. Xian Y, Xu H, Lytle B, Blevins J, Peterson ED, Hernandez AF, et al. Use of Strategies to Improve Door-to-Needle Times With 
Tissue-Type Plasminogen Activator in Acute Ischemic Stroke in Clinical Practice: Findings from Target: Stroke. Circulation Cardiovascular quality and outcomes. 2017;10 1; doi: 10.1161/circoutcomes.116.003227.

19. Man S, Zhao X, Uchino K, Hussain MS, Smith EE, Bhatt DL, et al. Comparison of Acute Ischemic Stroke Care and Outcomes Between Comprehensive Stroke Centers and Primary Stroke Centers in the United States. Circulation Cardiovascular quality and outcomes. 2018;11 6:e004512; doi: 10.1161/circoutcomes.117.004512.

20. Menon BK, Xu H, Cox M, Saver JL, Goyal M, Peterson E, et al. Components and Trends in Door to Treatment Times for Endovascular Therapy in Get With The Guidelines-Stroke Hospitals. Circulation. 2019;139 2:169-79; doi: doi:10.1161/CIRCULATIONAHA.118.036701.

21. Tran D, Zhu Z, Shafie M, Abcede H, Stradling D, Yu W. Three easily-implementable changes reduce median door-to-needle time for intravenous thrombolysis by 23 minutes. BMC Neurol. 2019;19 1:300; doi: 10.1186/s12883-019-1527-8.

22. Schwamm LH, Fonarow GC, Reeves MJ, Pan W, Frankel MR, Smith EE, et al. Get With the Guidelines-Stroke is associated with sustained improvement in care for patients hospitalized with acute stroke or transient ischemic attack. Circulation. 2009;119 1:10715; doi: 10.1161/circulationaha.108.783688.

23. Frei D, McGraw C, McCarthy K, Whaley M, Bellon RJ, Loy D, et al. A standardized neurointerventional thrombectomy protocol leads to faster recanalization times. Journal of neurointerventional surgery. 2017;9 11:1035-1040; doi: 10.1136/neurintsurg-2016012716 .
24. Psychogios MN, Behme D, Schregel K, Tsogkas I, Maier IL, Leyhe JR, et al. One-Stop Management of Acute Stroke Patients: Minimizing Door-to-Reperfusion Times. Stroke. 2017;48 11:3152-3155; doi: 10.1161/strokeaha.117.018077.

25. Ribo M, Boned S, Rubiera M, Tomasello A, Coscojuela P, Hernández D, et al. Direct transfer to angiosuite to reduce doorto-puncture time in thrombectomy for acute stroke. Journal of neurointerventional surgery. 2018;10 3:221-224; doi: 10.1136/neurintsurg-2017-013038.

26. Kamal N, Holodinsky JK, Stephenson C, Kashayp D, Demchuk $\mathrm{AM}$, Hill MD, et al. Improving Door-to-Needle Times for Acute Ischemic Stroke: Effect of Rapid Patient Registration, Moving Directly to Computed Tomography, and Giving Alteplase at the Computed Tomography Scanner. Circulation Cardiovascular quality and outcomes. 2017;10 1; doi: 10.1161/circoutcomes.116.003242.

27. Khatri P, Kleindorfer D, Devlin T, et al. Alteplase for the treatme nt of acute ischemic stroke in patients with low NIHSS and not clearly disabling defic its: Primary results of the PRISMS trial. Stroke 2018;49:LB9. Presented at: ISC 2018. J an. 25, 2018. Los Angeles.

28. Khatri P, Kleindorfer DO, Devlin T, Sawyer RN, Jr., Starr M, Mejilla J, et al. Effect of Alteplase vs Aspirin on Functional Outcome for Patients With Acute Ischemic Stroke and Minor Nondisabling Neurologic Deficits: The PRISMS Randomized Clinical Trial. Jama. 2018;320 2:156-166; doi: 10.1001/jama.2018.8496.
This work is licensed under Creative Commons Attribution 4.0 License

To Submit Your Article Click Here: Submit Manuscript

DOI: $10.31579 / 2578-8868 / 184$
Ready to submit your research? Choose Auctores and benefit from:

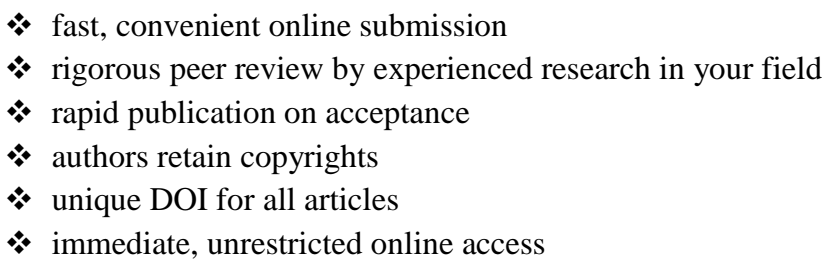

At Auctores, research is always in progress.

Learn more www.auctoresonline.org/journals/neuroscience-andneurological-surgery 